Original Research Article

\title{
Tear Film Break up time (TBUT) in allergic papillary conjunctivitis and immediate effect of carboxy methylcellulose on TBUT
}

\author{
Jarmale R. ${ }^{1}$, Jagadeeshwara V.M. ${ }^{2}$ \\ ${ }^{1}$ Dr. Rathnamma Jarmale, ${ }^{2}$ Dr. Vidhya Mysore Jagadeeshwara, Assistant Professor, Department of Ophthalmology, Subbaiah \\ Institute of Health Sciences, Purule, Shimoga, Karnataka, India.
}

Corresponding Author: Dr.Vidhya Mysore Jagadeeshwara, Assistant Professor, Department of Ophthalmology, Subbaiah Institute of Health Sciences, Purule, Shimoga, Karnataka, India. Email: mjvidya_doc@yahoo.co.in

\begin{abstract}
Purpose: To find out the effect of papillae due to allergic conjunctivitis on the tear film break up time (TBUT) and immediate effect of topical carboxymethyl cellulose (CMC) on TBUT in these patients. Methods: 62 eyes of the 31 patients in the age group of 20-40 years with papillae in the tarsal conjunctiva due to allergic conjunctivitis were subjected to measurement of TBUT. This was followed by application of one drop of $0.5 \%$ carboxy methylcellulose eye drop and TBUT was repeated after 5 minutes. Patients not in the 20-40-year age group, presence of any ocular surface disorder, mebomian gland dysfunction and lagophthalmos were excluded from the study. Results: 31 patients (62 eyes) were included in the study. TBUT was less than $15 \mathrm{secs}$ in 47 eyes $(75.80 \%)$ and more than $15 \mathrm{sec}$ in 15 eyes $(24.19 \%)$. TBUT improved after application of topical CMC in 23 eyes $(37.09 \%)$ and reduced in 21 eyes $(33.87 \%)$ and remained the same in 18 eyes $(29.56 \%)$. Percentage of patients who showed improvement in TBUT who had less than normal TBUT was $40.42 \%$. Percentage of patients who showed reduction in TBUT with normal TBUT was $66.66 \%$. Conclusion: TBUT is less than normal in around two third of the patients with allergic papillary conjunctivitis. Immediate effect of topical CMC showed no significant changes in the TBUT. Topical CMC drops showed better improvement in TBUT in patients with initial reduced TBUT rather than in patients with normal initial TBUT.
\end{abstract}

Keywords: Allergic papillary conjunctivitis, TBUT, Topical carboxymethylcellulose

\section{Introduction}

Tear film break up time is the time required for tear film to lose cohesive surface wetting after each blink. TBUT is useful in assessing the evaporative type of dry eye disease. It provides the important measurement of protective action of the tears. Dry areas appear due to normal evaporation and diffusion of tears. In normal healthy eyes, dry areas appear between the blinks and further an urge to blink is triggered. When TBUT is less than blink rate the ocular surface is left unprotected and signs and symptoms of dry eye are accentuated. Average TBUT is considered around 15-20 seconds [1].

Ocular allergies usually coexist with other forms of allergies. Allergic conjunctivitis may be acute or chronic. Seasonal allergic conjunctivitis (SAC) and perennial allergic conjunctivitis (PAC) are generally milder than the chronic forms like atopic and vernal keratoconjunctivitis. In SAC and PAC the ocular inflammation is secondary to the $\mathrm{IgE}$ mediated hypersensitivity reaction. The mast cell activation and degranulation induce the inflammatory

Manuscript received: $8^{\text {th }}$ October 2019

Reviewed: $20^{\text {th }}$ October 2019

Author Corrected: $28^{\text {th }}$ October 2019

Accepted for Publication: $2^{\text {nd }}$ November 2019

Tropical Journal of Ophthalmology and Otolaryngology process in the conjunctival mucosa leading to the signs and symptoms of allergic conjunctivitis. Patients presents with redness, watering, itching and burning sensation in the eye. Examination of the eye may show conjunctival hyperaemia, mild papillary reaction, variable amount of chemosis and lid oedema.

Traditionally, allergic conjunctivitis and dry eye syndrome are regarded as two different diseases. Recent literature has shown both conditions share similar characteristics, including several signs and symptoms [2. Seasonal allergic conjunctivitis is associated with tear instability and thickening of the tear film lipid layer [3].

Activated T cells and an increase in inflammatory cytokines such as epidermal growth factor and interleukins damage the goblet cells and conjunctival epithelium which account for subsequent reduction in TBUT [4,5]. CMC is an anionic cellulose derivative, provides bio adhesive characteristics, and increases the tear retention time. It is also known for improving the health of conjunctival and corneal cells [6]. It alleviates the symptoms of dryness and irritation. Most of the patients receive topical lubricant along with 


\section{Original Research Article}

antihistamine or mast cell stabiliser as a prescription for the treatment of allergic papillary conjunctivitis. The study is directed to know whether all the patients with allergic papillary conjunctivitis benefit with topical carboxymethyl cellulose. The present study aims at identifying the changes in TBUT in patients with allergic papillary conjunctivitis and the immediate effect of topical carboxy methylcellulose on TBUT.

\section{Materials and Methods}

\section{Study description}

Setting: The subjects of the present study were the patients who attended the outpatient department of ophthalmology in Subbaiah Institute of Medical College, Shimoga, India for either investigation and/ or treatment of ocular disorders and met the inclusion criteria.

Study type and duration: It is a cross sectional observational study (clinical trial) carried out during the month of November 2018 .

Sampling methods: The participants in the study were chosen by convenient sampling. 62 eyes of 31 patients were included in the study. Sample size was calculated based on the Wilcoxon Signed Rank test.

\section{Inclusion criteria}

- Age - 20-40 years

- Sex - all

- Signs and symptoms suggestive of allergic conjunctivitis

- Presence of mild papillary reaction in the upper tarsal conjunctiva

\section{Exclusion criteria}

- Contact lens wearers

- Blepharitis

- Mebomian gland dysfunction

- Lid abnormalities

- Corneal or conjunctival scarring

- History of using any topical eye medications

- Presence of systemic diseases like rheumatoid arthritis, scleroderma

Data collection procedure: The aim and procedure of the study was explained to each subject. All the subjects were enquired regarding the symptoms experienced, any history of using topical eye drops previously and usage of contact lenses. Examination of the patient's eyes was carried out with slit lamp.

Conjunctiva was examined for the presence of papillae in the upper tarsal region. Any signs suggestive of ocular surface disorder, mebomian gland dysfunction, lid abnormalities were looked for.

Patients with symptoms and signs suggestive of allergic conjunctivitis and with papillae in the upper tarsal conjunctiva were subjected to measurement of TBUT. Tear film was stained with moist sterile Fluorescein strips and patient was asked to blink a couple of times to spread the dye.

Tear film was examined under the slit lamp using cobalt blue filter. Patient was advised to restrain from blinking during the process of calculating TBUT.

Number of seconds that elapse between the last blink and the appearance of first dry spot in the tear film was recorded as the TBUT. This was followed by application of one drop of $0.5 \%$ carboxy methylcellulose eye drop and TBUT was repeated after 5 minutes of application of topical carboxy methylcellulose drops.

Same procedure was repeated in the other eye. All patients were applied the same concentration and the same brand of carboxymethyl cellulose drops. 
Original Research Article

Data Analysis: The non parametric test - Wilcoxon signed rank test was used for data analysis.

\begin{tabular}{|c|c|c|c|}
\hline Group & N & Median (IQR) & P value \\
\hline Initial TBUT & 62 & $9(6,15.25)$ & \\
\hline TBUT after lubricant eye drop application & 62 & $10(6,19)$ & 0.446 \\
\hline
\end{tabular}

Ethical Consideration and permission: The study protocol was approved by the ethical committee of Subbaiah Institute of Medical Sciences

\section{Results}

Out of 31 patients $9(29.04 \%)$ were male and $22(70.96 \%)$ were female. TBUT was less than normal ( $<15$ seconds) in 47 $(75.80 \%)$ and normal in $15(24.20 \%)$ out of 62 eyes (Table 1$)$.

Table-1: Demographic characteristics of study patients.

\begin{tabular}{|c|c|c|c|}
\hline Age group (years) & Number of patients & Male & Female \\
\hline $20-24$ & 8 & 2 & 4 \\
\hline $25-29$ & 6 & 2 & 7 \\
\hline $30-34$ & 9 & 3 & 5 \\
\hline $35-40$ & 8 & 2 & 6 \\
\hline
\end{tabular}

TBUT was considered to be improved if there was an increase of 2 seconds or more from the baseline value and reduced if the TBUT decreased by 2 or more seconds from the baseline value. TBUT improved in 23 eyes after application of topical CMC eye drops. Out of 23 eyes, 19 eyes had initial TBUT below normal and 4 eyes were above normal. Improvement in TBUT after application of topical CMC was more in males compared to females. In $40.9 \%$ of females the TBUT reduced from the baseline value after application of topical CMC. Table 2 shows the details of the changes in TBUT after application of topical CMC.

Table-2: Changes in TBUT after application of topical CMC eye drops

\begin{tabular}{|c|c|c|}
\hline TBUT & Males (9) & Females (22) \\
\hline Improved & $6(66.67 \%)$ & $4(18.19 \%)$ \\
\hline Reduced & $1(11.11 \%)$ & $9(40.90 \%)$ \\
\hline Same & $2(22.22 \%)$ & $3(13.64 \%)$ \\
\hline Differed in two eyes & 0 & $6(27.27 \%)$ \\
\hline
\end{tabular}

Around $40 \%$ of the patients with initial below normal TBUT showed improvement in the tear film stability post application of the topical CMC and it did not make a difference in the TBUT in around 36\% of patients. Further reduction of TBUT was seen in around $23 \%$ of patients (Table 3 ).

Table-3: Changes in TBUT in patients with initial below normal TBUT

\begin{tabular}{|c|c|c|}
\hline TBUT & Number of eyes & Percentage \\
\hline Improved & 19 & 40.42 \\
\hline Reduced & 11 & 23.40 \\
\hline Same & 17 & 36.17 \\
\hline
\end{tabular}

Around $66 \%$ of patients with initial normal TBUT showed reduction after application of topical CMC. In around $26 \%$ of patients there was improvement and in $6 \%$ it remained the same. As evident from the above tables the improvement of the TBUT is better in patients with below normal initial TBUT than with initial normal ones. The reduction of TBUT post application of topical CMC is greater in patients with initial normal TBUT (Table 4). 
Original Research Article

Table-4: Changes in TBUT in patients with initial normal TBUT

\begin{tabular}{|c|c|c|}
\hline TBUT & Number of eyes & Percentage \\
\hline Improved & 4 & 26.67 \\
\hline Reduced & 10 & 66.67 \\
\hline Same & 1 & 6.66 \\
\hline
\end{tabular}

\section{Discussion}

Allergic conjunctivitis includes a wide spectrum of clinical entities and is one of the frequent ocular surface disorders encountered in the clinical practice. SAC is the commonest form of allergic conjunctivitis due to exposure to allergens like pollen, grass and outdoor molds. Papillae represent the vascular changes seen in the palpebral conjunctiva where fibrous septae anchor conjunctiva to the tarsus. Papillae are commonly associated with allergic immune response, as in simple allergic and vernal keratoconjunctivitis or in response to a foreign body such as contact lens or ocular prosthesis.

Ocular allergies are associated with tear film instability, goblet cell loss and conjunctival squamous metaplasia. In addition to the presence of ocular surface inflammation, ocular allergy may cause damage to the eye through mechanical tear film instability [7]. Irregular ocular surface due to papillae in the conjunctiva is one of the factors contributing to the mechanical tear film instability.

There are also evidences of thickening of the lipid layer of the tear film and increased instability of the tear film in allergic conjunctivitis [3]. Unstable tear film exposes the ocular surface to further damage in addition to those caused due to allergic changes. The present study demonstrated an alteration of tear film in the form of reduced TBUT in about $75 \%$ of the patients having allergic papillary conjunctivitis.

Allergic eye disease has no universally accepted protocols of treatment. The primary goal of the treatment of allergic conjunctivitis is to reduce the inflammation and prevent complications which can threaten vision or can cause dry eye. The drugs commonly used in the treatment are topical antihistamines, mast cell stabilisers, lubricants and in severe cases steroids. Treatment with topical eye drops should be started immediately and continued for a period until all the symptoms have disappeared. Efforts should be made to prevent conversion of acute inflammation to a chronic one. Ocular lubricating eye drops are used in allergic conjunctivitis to dilute the inflammatory agents in tears

and to wash away the allergens to reduce the itching and further worsening of symptoms. Benzalkonium chloride in the topical drops may itself cause allergy and can worsen the already existing dry eye [8]. A study has observed that the reduced goblet cell density and conjunctival inflammation are exacerbated by the usage of topical agents with preservatives [9]. Antihistamines are also not suitable for prolonged usage as they too can increase the severity or even cause dry eye [8].

Hence lesser the number of topical drops with preservatives used better chances of improving the goblet cell density in the conjunctiva. Olopatidine, dual action antihistamine and mast cell stabiliser was shown to improve tear function and ocular surface status with regard to squamous metaplasia and loss of goblet cells [10].

Topical carboxymethycellulose is commonly available in the concentration of $0.5 \%$ and $1 \%$ ophthalmic solution. They can be preservative free or with preservatives. Conventionally it is used in the treatment of dry eye disease. On long term usage it is shown to improve conjunctival and corneal staining sum scores, tear film breaks up time and dry eye symptoms [11-13].

The present study showed that there is no immediate significant change in the TBUT in patients with allergic papillary conjunctivitis after application of topical CMC. Even though there is improvement in the TBUT, it was more evident in the patients having lower initial TBUT than in the patients with initial normal TBUT.

Topical CMC eye drops in allergic papillary conjunctivitis may not be helpful in all patients. The validation of usage of topical CMC in allergic conjunctivitis patients has to be preceded by the evidence of dry eye in them.

Unnecessary prescription of topical $\mathrm{CMC}$ in allergic conjunctivitis is not only an economic burden on the patient but also the preservatives used in the drops may incite the dry eye. Even though preservative free medications are available, they are again an economic constraint to the patient. Drugs like Olopatidine which has evidence of improving the ocular surface health along with the reduction of ocular inflammation caused by allergy may be preferred instead of adding multiple topical applications. To summarise, allergic papillary conjunctivitis causes an alteration in the tear film and the immediate effect of topical 
Original Research Article

CMC shows no significant changes in TBUT. But the group of patients having less than normal initial TBUT had better response to topical CMC than normal initial TBUT.

Primary analysis of TBUT before prescribing the topical lubricant drops in patients with allergic conjunctivitis may be helpful in avoiding unnecessary prescription of the drug.

The limitations of the study are its smaller sample size. It studies only the immediate effect of topical lubricant eye drops on the TBUT in allergic papillary conjunctivitis. Further studies are needed to analyse the long-term effect of topical lubricants on TBUT with larger sample size.

\section{Conclusion}

TBUT is less than normal in around two thirds of the patients with allergic papillary conjunctivitis. Immediate effect of topical CMC showed no significant changes in the TBUT. Topical CMC drops showed better improvement in TBUT in patients with initial reduced TBUT rather than in patients with normal initial TBUT.

Prescription of topical carboxy methylcellulose in allergic papillary conjunctivitis should be preceded by simple TBUT examination to analyse the efficacy of the topical lubricant drops in the patient.

\section{What the study adds to the existing knowledge}

No significant immediate change in the TBUT in patients with allergic conjunctivitis after usage of topical carboxymethylcellulose.

\section{Author's contribution}

Dr. Rathnamma J: Verified the analytical methods and supervised the findings of the work.

Dr. Vidhya M J: Conceived the idea and performed the procedure and data collection.

Both the authors discussed the final results and prepared the manuscript.

\section{Acknowledgements}

This research was supported by Subbaiah Institute of Health Sciences, Shivamogga. Authors thank all the faculty in the Ophthalmology department and Research department of the institution for their contribution in preparing the manuscript.

Funding: No funding sources

Conflict of interest: None declared

Ethical approval: This study was approved by the Institutional Ethics Committee

\section{References}

1. Phadatare SP, Momin M, Nighojkar P, Askarkar S, Singh KK. A comprehensive review on dry eye disease: Diagnosis, medical management, recent developments and future challenges. Advan Pharmaceut.2015. doi:10. 1155/ $2015 / 704946$.

2. Fujishima H, Toda I, Shimazaki J. Allergic conjunctivitis and dry eye. Br J Ophthalmol. 1996; 80 (11): 994-997. doi:10.1136/bjo.80.11.994.

3. Suzuki, Shintaro MD,Goto. Tear film lipid layer alteration in allergic conjunctivitis. J Cornea Ext Dis. 2006; 25 (3): 277-280.doi:10.1097/01.ico.0000178277. 29010.bc.

4. Dogru M, Katakami C, Nakagawa N. Impression cytology in atopic dermatitis. Ophthalmol. 1998:105(8): 1478-1484. doi: https://doi.org/10.1016/S0161-6420 (98 ) 98032-0.

5. Dogru M, Okada N, Asano Kato N. Atopic ocular surface disease:Implication on tear function and ocular surface mucin. Cornea. 2005;24 (8):518-523.doi:10. 1097/ 01.ico. 0000178741.14212 .53 .

6. Garrett Q, Simmons PA, Xu S, Vehige J, Zhao Z, Ehrmann $\mathrm{K}$ et al. Carboxymethylcellulose binds to human corneal epithelial cells and is a modulator of corneal epithelial wound healing. Investi Ophthalmol Vis Sci. 2007, 48:1559-1567.doi:10.1167/iovs.06-0848.

7. Andrea Leonardi, Anthony Hall. Mechanisms of corneal allergic reaction: New options for treatment. Expert Rev Ophthalmol. 2010;5(4):545-556.

8. Osmo Kari, K Matti Saari. Updates in the treatment of ocular allergies. J Asthma Allergy. 2010:3:149-158. doi: 10.2147/JAA.S13705.

9. Julie. M. Albietz, Adrian. S. Bruce. The conjunctival epithelium in dry eye subtypes: effect of preserved and nonpreserved topical treatments. Current Eye Res. 2009; 22(1): 8-18.doi:10.1076/ceyr.22.1.8.6977.

10. Dogru M, Ozmen A, Erturk H. Changes in tear function and the ocular surface after topical olopatidine treatment for allergic conjunctivitis: an open case study. Clinic Therapeut. 2002; 24(8):1309-1321. doi:10.1016/ S01492918 (02) 80035-2.

11 Lee JH, Ahn HS, Kim E, Kim T. Efficacy of sodium hyaluronate and carboxymethylcellulose in treating mild to moderate dry eye disease. Cornea: 2011;30(2):175-179. doi:10.1097/CO.0b013e3181e9adcc. 


\section{Original Research Article}

12. Yao K, Boa Y, Yang J, Lu Y. Efficacy of $1 \%$ carboxymethylcellulose sodium for treating dry eye after phacoemulsification: results from a multicentre, open label randomized, controlled study. BMC Ophthalmol. 2015; 15:28. doi:10.1186/s12886-105-0005-3.
13. Peter AS, Joseph V. Clinical performance of a midviscosity artificial tear for dry eye treatment. Cornea. 2007; 26 (3): 294-302. doi: 10.1097/ICO0b013e31802 e1e04.

\section{How to cite this article?}

Jarmale R, Jagadeeshwara V.M. Tear Film Break up time (TBUT) in allergic papillary conjunctivitis and immediate effect of carboxy methylcellulose on TBUT. Trop J Ophthalmol Otolaryngol.2019; 4(7):403-408. doi:10.17511/jooo.2019.i07.01 